\title{
Institutionnalisme et développement : les performances des institutions scolaires
}

\section{Philippe Hugon}

\section{Q OpenEdition}

12 Journals

Édition électronique

URL : http://journals.openedition.org/ei/5644

DOI : $10.4000 /$ ei.5644

ISSN : 2553-1891

Éditeur

Association Économie et Institutions

Référence électronique

Philippe Hugon, « Institutionnalisme et développement : les performances des institutions scolaires », Économie et institutions [En ligne], 20-21 | 2014, mis en ligne le 01 juin 2014, consulté le 30 avril 2019. URL : http://journals.openedition.org/ei/5644; DOI : 10.4000/ei.5644

Ce document a été généré automatiquement le 30 avril 2019

Revue Économie et institutions 


\title{
Institutionnalisme et développement : les performances des institutions scolaires
}

\author{
Philippe Hugon
}

\section{Introduction}

1 La performance est au cœur de la représentation universaliste de l'économie standard en termes d'efficience et d'efficacité, de rationalisation et de gestion de la rareté. Elle implique la construction de cadres de cohérences qui ont pour effet de rendre les informations homogènes. Le développement économique est assimilé à des institutions plus efficientes et plus spécialement à des progrès de l'éducation, qui est porteuse de rationalité. Les modèles néo classiques, post keynésiens ou de la croissance endogène convergent sur ces propositions. Cette représentation dominante a conduit à mettre l'accent sur les institutions et les réformes institutionnelles

2 En réalité, les institutions diffèrent selon les sociétés et les critères de performance sont pluriels et les thèses universalistes de l'institution scolaire reposent sur une conception socialement désincarnée. Il y a multidimensionnalité des performances de l'éducation dans le développement. Dans toutes les sociétés, la performance tient aux conventions arbitraires qui la fondent (Boltanski, Thevenot 1991), au fait que l'évaluation des coûtsavantages ne peut être dissociée du pouvoir qu'elle implique (Bachelard, 1973). Tout pouvoir cherche à réduire la complexité et la diversité du social en un ordre unifié et manipulable (Foucault 2001). La pluralité des critères de performance se pose avec une acuité particulière dans les sociétés du Sud ou en développement.

3 Les résultats des réformes institutionnelles et les références théoriques et avancées dans la première partie permettent de mieux rendre en compte l'hétérogénéité et de la conflictualité liées aux processus éducatifs dans les pays en développement. Cet article après avoir présenté : (1) la question générale de la pluralité des performances des 
institutions dont l'institution éducative dans les sociétés en développement et montré la pertinence d'une économie politique institutionnelle (2) analysera plus spécifiquement les liens entre institutions éducatives et développement

\section{Performance des institutions et économie politique institutionnelle}

4 Alors que les modèles économiques standards faisant abstraction des institutions ont longtemps dominé, le rôle des institutions et des réformes institutionnelles est devenu aujourd'hui central dans les pays en développement. Le développement des compétences, des connaissances, des capacités et potentialités des hommes que permet l'éducation est considérée comme étant au cœur du processus de développement des capacités et des potentialités des sociétés. Dans ces sociétés, la question de la performance des institutions se pose toutefois de manière spécifique Elle ne peut être dissociée des contextes de relations asymétriques et des modes d'appropriation des greffes institutionnelles. Nous montrerons dans cette première partie que les limites des réformes institutionnelles dans les pays du Sud (1.1) obligent à revisiter les diverses théories institutionnalistes (1.2) pour comprendre la diversité des formations institutionnelles dans un contexte aujourd'hui mondialisé (1.3).

\subsection{Réformes et normes institutionnelles universelles}

5 Les institutions en général et scolaires en particulier sont généralement situées au cœur du processus des transformations sociales, des systèmes de représentation et du passage de sociétés traditionnelles à des sociétés modernes et innovantes ou des ordres sociaux d'accès limité à des sociétés d'accès ouvert (North et al. 2006). Ainsi North et al. (2006) reprenant la tradition d'une représentation dualiste et évolutionniste différencient les sociétés d'accès ouverts (se référant au modèle idéal occidental de démocratie et de marché) et les sociétés d'accès limités où les élites prélèvent des rentes en assurant la stabilité. La régulation de la violence dans des États naturels se fait par une manipulation politique de l'économie visant à établir rentes et privilèges ; l'école et les institutions éducatives sont au cœur de cette reproduction des hiérarchies dans un système d'accès fermé. Dans un ordre social d'accès ouvert, l'école universelle comme les partis politiques, les entreprises, les syndicats les medias sont au cœur des transformations conduisant au décollage économique. Les causes ultimes du développement se trouvent, dès lors, dans les institutions qui génèrent des comportements humains, structurent les incitations, modèlent les croyances et légitiment les règles.

\subsubsection{Les indicateur de performance des institutions}

6 La performance ou la qualité des institutions est devenue aujourd'hui un des critères majeurs pour caractériser les économies en développement et préconiser des réformes de la part des organisations internationales. La hiérarchie par des notes des élèves de la classe internationale permet de différencier les «bons élèves" qui ont des bonnes performances et ceux qui sont en situation d'échec ou de progrès. Les premiers instruments de mesure ont été le PIB proxy de l'efficience économique. Puis les indicateurs du développement humain préconisés par le PNUD ont pris en compte l'équité sociale et 
introduit la variable scolaire. Les indicateurs ont ensuite intégré les questions de soutenabilité écologique (empreinte écologique) et les indicateurs politiques de liberté, de stabilité, de « bonne gouvernance » et de sécurité.

7 Les principaux critères de classement sont devenus aujourd'hui les indicateurs institutionnels. Cette volonté d'établir des indicateurs traduit la volonté de la recherche de performance, et d'efficacité, de management par la qualité résultats d'un libéralisme normalisateur, d'où la priorité accordée à l'évaluation (accountability). La qualité institutionnelle est mesurée par des indicateurs de démocratie (participation des citoyens, responsabilisation, stabilité politique, absence de violence), de capacité de gouvernement (efficacité des pouvoirs publics, poids des règlementations), du respect des citoyens et de l'État envers les institutions qui gouvernent les relations économiques et sociales (État de droit, absence de corruption). Elle se définit en référence au marché : la protection des droits de propriété, la réglementation, la stabilisation des marchés et la légitimation des marchés (Rodrik, Subramanian 2004).

\subsubsection{Les réformes institutionnelles et la difficile mise en place des « bonnes » institutions.}

Les responsables des pays en développement mettent ainsi en place, sous l'impulsion des bailleurs de fonds et des institutions de Bretton Woods, des réformes institutionnelles. Elles visent à favoriser le passage d'économies administrées à des économies de marché, de régimes autocratiques à des régimes démocratiques et d'État de non droit à des États de droit, à protéger les droits de propriété. Il y a débat quant au rythme souhaitable des réformes (réformes graduelles versus «big bang »), quant aux séquences (réformes des institutions économiques versus institutions politiques). Pourquoi existe-t-il de «mauvaises» institutions alors que selon North l'histoire sélectionne les meilleures? Deux réponses sont possibles. Soit, il existe des référents socio culturels et des systèmes de valeurs de représentation s'opposant à des institutions efficientes. Il faut alors, dans la tradition du culturalisme voire du relativisme, analyser les conflits entre rationalité économique et logiques sociales, religieuses ou culturelles. Soit, les autorités politiques ont intérêt à choisir des institutions inadéquates. Il faut alors remonter aux logiques de pouvoirs.

Les réformes éducatives et l'amélioration de leurs performances sont au cœur de ces transformations institutionnelles. Il importe de passer d'écoles traditionnelles à des écoles innovantes fondées sur les initiatives et la compétition .Les écoles ou les universités privées et payantes sont généralement considérées comme étant plus incitatives vis-à-vis des parents et des élèves. Il importe d'améliorer la pyramide scolaire en visant à généraliser l'éducation de base et en régulant les flux du secondaire et du supérieur évitant les universités parking. Les systèmes éducatifs doivent être mis au regard d'évaluation et de notation permettant de comparer leurs performances au niveau international.

Les travaux les plus sophistiqués à partir de batteries d'indicateurs tels ceux de Meisel et Ould-Aoudia (2007) montrent que la « bonne gouvernance » est corrélée avec le niveau du PIB par tête mais non avec sa croissance. Il importe, dès lors de mettre en relation les changements institutionnels à partir d'indicateurs de profils institutionnels avec les indicateurs de décollage et de convergence des économies. Le développement apparait alors comme un processus de changement institutionnel de long terme caractérisé par 
une dépersonnalisation des systèmes des relations sociales, des degrés accrus de formalisation et de respect des règles légitimées, des régulations des jeux conflictuels entre les groupes d'intérêts et de la violence, une vision stratégique à long terme des décideurs, une confiance réductrice des incertitudes, une aptitude de la société à l'innovation, la concertation pour faire émerger le bien commun, la qualité des biens et services publics, la sécurité des transactions et des droits fonciers.

\subsubsection{Les limites des tests sur les performances des institutions}

11 Ces approches normatives en termes de "bonnes institutions" reposent sur une vision téléonomique et universaliste des sociétés et sont une manière de réactualiser l'opposition entre sociétés traditionnelles et sociétés modernes. Elles conduisent le plus souvent à transplanter des greffes institutionnelles au lieu de prendre en compte les spécificités des contextes des sociétés et les trappes à pauvreté ou à conflits. Les résultats de ces réformes institutionnelles sont ainsi contrastés. De nombreuses réformes institutionnelles ont échoué car elles remettaient en cause les fragiles équilibres socio politiques et même ont parfois accentué la conflictualité. Une réforme comme la privatisation ou la libéralisation des prix peut être rationnelle économiquement. Elle peut également remettre en question les équilibres régionaux et sociopolitiques qui rétro agissent sur la sécurité et donc sur la prise de risque de l'investissement. La plupart des tests économétriques se situent dans une perspective synchronique en mettant en relation des indicateurs statiques de qualité institutionnelle et les indicateurs de performances économiques. Ces travaux n'échappent pas aux biais d'une vision normative voire téléologique. Ils réduisent les rapports sociaux et rapports de pouvoir à des indicateurs normés et politiquement correct. Ils traitent de même manière les micro nations ou quasi États et les grandes puissances. Ils isolent les institutions au lieu d'analyser la pluralité des profils et configurations institutionnels et leur hybridation.

\subsection{Retour sur la pluralité des institutions et les débats institutionnalistes}

Un retour sur les diverses théories institutionnalistes est ainsi nécessaire pour prendre en compte la pluralité des institutions et les différentes manières $d$ ' analyser leurs performances Les courants institutionnalistes se situent sur le curseur allant de l'unité des calculs des arrangements institutionnels des agents à la diversité des stratégies des acteurs et d'une vision synchronique des coordinations et celle diachronique des régulations et conflits (Billaudot, 2004 ; Hall et Taylor,1997; Tallard, Theret, Uri, 2000)

Tableau 1. Typologie des institutionnalismes selon le rapport au temps et à la rationalité et diversité des critères de performances

\begin{tabular}{|c|c|c|}
\hline $\begin{array}{ll}\text { Rapport } & \text { au } \\
\text { Temps/à } & \text { la } \\
\text { rationalité } & \end{array}$ & Synchronie (coordination) & Diachronie (conflits/ régulation) \\
\hline Unité des calculs & $\begin{array}{l}\text { Institutionnalisme rationnel } \\
\text { Unicité des performances }\end{array}$ & $\begin{array}{l}\text { Institutionnalisme historique, fruit des } \\
\text { rapports sociaux, évolution des } \\
\text { performances }\end{array}$ \\
\hline
\end{tabular}




\begin{tabular}{|l|l|l|}
\hline $\begin{array}{l}\text { Diversité des } \\
\text { stratégies }\end{array}$ & $\begin{array}{l}\text { Institutionnalisme } \\
\text { conventionnaliste Pluralité des } \\
\text { critères de performances }\end{array}$ & $\begin{array}{l}\text { Économie politique institutionnaliste } \\
\text { Pluralité des architectures et des } \\
\text { formations institutionnelles }\end{array}$ \\
\hline
\end{tabular}

13 L'institutionnalisme rationnel et individualiste de Coase (1988), North (1990) et Williamson (2000) traite des institutions en termes d'efficience, de capacité à réduire les coûts de transaction ou de règles du jeu (Aoki 2005). Elles comprennent selon North (1990) des contraintes informelles, telles que les coutumes ou les codes de comportement, et des règles formelles, comme les lois ou les droits de propriété. Selon Aoki (2005), une institution est un système autoentretenu de croyances partagées sur la manière dont le jeu est joué ; elle combine des joueurs (organisations), des règles du jeu et des équilibres pouvant être formalisés par la théorie des jeux. L'économie néo-institutionnelle s'est développée autour de trois principaux postulats (He Yong, 1994): les institutions sont déterminantes dans l'allocation des ressources; elles peuvent être traitées de manière endogène ; elles ont un fondement micro économique dans le sens où l'on peut expliquer des choix organisationnels. Selon ce courant, les institutions concernent les régularités du comportement social convenu par tous les membres de la société. Dans le cas d'informations asymétriques, des substituts au marché apparaissent sous forme de relations hiérarchiques ou de contrats, ceux-ci limitent les coûts de transaction. Ces modes de coordinations facilitent en outre l'action collective des acteurs privés ou publics, qu'une coordination purement marchande ne peut réaliser. Les institutions joueraient enfin un rôle incitatif (North et Thomas, 1973) ont ainsi montré que la performance économique dans le monde occidental résultait prioritairement de la création de dispositifs institutionnels et de droits de propriété stimulant l'effort économique privé. Selon cette approche, les institutions scolaires peuvent être analysées au regard de leur efficience et du choix rationnel des divers modes de coordination privé ou publique notamment par leur rôle incitatif sur l'apprentissage des savoirs.

14 L'institutionnalisme sociologique, cognitif ou psychologique (ex des conventions ou des interactions sociales) réduit également les institutions à des modes de coordination mais elle prend en compte la pluralité des espaces de justification. Le cognitivisme analyse les processus mentaux par lesquels les agents forment leurs représentations imparfaites $\mathrm{du}$ monde. Les institutions ne sont pas seulement des faits et des pratiques collectives mais aussi des cadres cognitifs et moraux dans lesquels se développent des pensées individuelles (Douglas 2004). Les conventions sont ainsi des moyens de coordination arbitraires nécessaires à des individus rationnels ayant des intérêts communs, des interprétations liées à des incomplétudes de règles, qui améliorent l'efficacité coopérative des acteurs concernés. Il y a selon les espaces de légitimation ou cités ou les conventions plusieurs critères possibles de performances (Boltanski, Thevenot 1991, Favereau 1995, Boltanski, Chiapello 1999). Les institutions scolaires peuvent être analysées au regard des cités inspirées (imagination, écoles d'artistes), civiques (école républicaine), sacrée (école religieuse), domestique (école traditionnelles reproduisant les hiérarchies), cité de l'opinion (réputation), cités marchandes (école avec concurrence et rivalité), cité industrielle (école scientifiques d'ingénieur avec efficacité), cité par projets (école avec autonomie et employabilité).

15 L'institutionnalisme historique prend en compte selon Billaudot (2004) les conflits sociaux comme rendant compte de processus historique et d'une approche qui part des objets 
collectifs. L'institutionnalisme américain, en particulier à Commons en est un des fondateurs. Plusieurs écoles de pensée peuvent être regroupées sous ce «label». L' évolutionnisme s'intéresse à la genèse et à l'évolution liée à un processus de sélection (Nelson, Winter 1982) des institutions liées aux rapports sociaux, au caractère irréversible $\mathrm{du}$ temps du développement et à leur dimension politique. Les institutions sont instituées, évolutives et créées tout à la fois. Elles sont le fruit d'une histoire et des construits sociaux. Cette conception historique réintroduit la violence et les conflits, occultés par les deux courants précédents (cf. la violence des droits de propriété liés aux enclosures, à la colonisation ou aujourd'hui aux transactions foncières dans le Sud). Les théories de la régulation visent, dans le cadre d'états-nations, à lier modes d'accumulation et formes institutionnelles. Malgré leur volonté d'élargir son champ aux économies en développement (Tr2), des différencier plusieurs formes de capitalismes ou de prendre en compte l'articulation de divers modes de production, elles demeurent largement des analyses théorie de l'encastrement politique des économies capitalistes bouclées dans le cadre d'États-nations avec le plus souvent références aux cinq formes institutionnelles canoniques (Boyer, Saillard 1995) L'institution scolaire est notamment analysée en relation avec le rapport salarial considéré comme référent.

L'économie politique institutionnaliste, à laquelle nous nous référons suppose, de prendre en compte la violence de la mise en contact de plusieurs institutions, l'hybridation des formations institutionnelles dans un contexte jadis colonisé et aujourd'hui mondialisé, avec des jeux de conflits, de ruses et d'accommodement créant des dynamiques de rejet ou des greffes institutionnelles. Elle suppose ainsi d'analyser la légitimité des institutions, leurs spécificités et la place qu'elles occupent au sein de l'architecture institutionnelle les réformes institutionnelles. Prendre en compte les conflits générés par les réformes institutionnelles est stratégique pour une appropriation des réformes, et une endogénéisation des institutions (Hugon 2012). C'est leur complémentarité qui fait système (Lafaye de Micheaux, Ould-Ahmed 2007). Les institutions qui comptent n'ont pas pour origine un principe économique d'efficience. Les formes institutionnelles et la codification des rapports sociaux fondamentaux supposent de remonter aux rapports de pouvoir et aux rapports sociaux. Les arrangements institutionnels renvoient à l'ensemble des règles et comportements qui gouvernent les actions et les relations entre les individus ; ils peuvent être à la fois formel (familles, firmes, hôpitaux, universités, etc.) ou informel (valeurs, coutumes, traditions, etc.); ils répondent à la fois à une fonction de réduction des coûts de transaction et des incertitudes. Les structures ou configurations institutionnelles ont, au contraire, une épaisseur sociétale et sont insérés dans des histoires longues et répondent à des contextes spécifiques (Yifu Lin, 1989). Cette approche, que nous développons dans la seconde partie, permet d'analyser comment des institutions scolaires importées ou imposées de l'extérieur ont conduit à des rejets, à des substitutions ou à des hybridations face aux institutions éducatives endogènes ou «traditionnelles ». Elle implique de prendre en compte diverses échelles allant du niveau local (ex des écoles primaires) au niveau mondial (ex des formations d'élites mondialisées) sans privilégier nécessairement l'échelle nationale en termes de « pays ».

\subsection{Universalité et spécificité des institutions}

La compréhension des formations institutionnelles suppose de différencier les institutions universelles ou universalisables, celles spécifiques à des sociétés et celles qui résultent d'hybridation ou de métissages. 

anthropologiques: produire de la confiance, réduire l'incertitude, réguler la violence, sécuriser les biens et les personnes, transmettre le savoir et la connaissance. Certaines ont acquis une relative universalité apprendre à lire, écrire et compter. Il y a dans toute société humaine une définition des inclus et des exclus. Les questions communes à toutes les sociétés humaines sont celles de l'arbitrage entre une efficience économique (recherche ou non de la meilleure adéquation des moyens aux fins), une équité sociale (acceptation ou non de la hiérarchie et des inégalités ou exclusions), la sécurité interne et externe, une soutenabilité écologique (conception écocentrée ou anthropocentrée) et une sécurité et stabilité politique. Les institutions permettent d'instituer c'est-à-dire .de faire passer à l'état d'ensembles organisées et d'activités légitimées des objets collectifs (peuples nations) et individuels. Les institutions politiques régulent les rapports sociaux et la violence. Les institutions religieuses et culturelles renvoient à des valeurs et à des croyances communes. Les institutions économiques permettent l'échange et la coordination de décisions plus ou moins décentralisées. Les institutions sont à la fois stables et évolutives selon des degrés différents selon les sociétés. Elles sont des contraintes qui facilitent la gestion des rapports humains dans leurs interactions économiques et sociales. Elles forment à cet effet un cadre réglementaire à l'intérieur duquel se fondent et peuvent se mouvoir les régularités comportementales qui, à leur tour vont déterminer à la fois leur émergence mais aussi leur évolution (Ménard 1990). Mais en même temps, les changements et les adaptations institutionnels sont nécessaires afin que les changements dans les préférences sociales, l'évolution technologique, les structures politiques et économiques internes ou externes s'accommodent à la structure des incitations qui reflète la demande des institutions.

Mais les horizons temporels et les priorités entre ces quatre objectifs économiques, sociaux, écologiques et politiques different selon les sociétés. Les formes institutionnelles, la hiérarchie et la légitimation des institutions diffèrent toutefois entre les sociétés sacrées renvoyant au monde des dieux et des traditions et les sociétés où leur légitimité renvoie aux contrats, aux conventions et au droit. Dans de nombreuses sociétés, les liens primaires (identités religieuses, ethniques, familiales ou tribales) l'emportent sur les liens secondaires de citoyenneté. Les institutions sont elles-mêmes évolutives et résultent d'une intégration de sociétés à petite échelle dans des sociétés plus larges généralement en raison de rapports de violence. Aujourd'hui, toutes les sociétés sont intégrées peu ou prou dans la société globale. La réalité est hybride et évolutive, faite de destruction/ restructuration, de combinaisons plus ou moins conflictuelles et de référents pluriels, de confrontation de systèmes de valorisation, de jeux d'acteurs dominants et dominés en situation d'interactions.

On peut parler ainsi dans les économies en développement, caractérisées par la violence des contacts avec les sociétés dominantes et l'insertion dans des interdépendances asymétriques, de formations institutionnelles et juridiques au sens des formations géologiques avec à la fois superposition et enchevêtrement des règles : droits coutumiers ou communautaires, droits issus des conquêtes (islamique, anglo-saxon, romano germanique,), droits sui generis des indépendances, droits issus des conditionnalités des Institutions de Bretton Woods. La colonisation n'est pas réductible à la transplantation d'institutions exogènes ou progressistes. Elle a conduit à une hybridation institutionnelle avec des jeux variables d'assimilation, de réinterprétation des règles ou de ruses. Les droits de propriété privée au cœur des débats du néo institutionnalisme n'ont qu'une 
légitimité limitée, dans les sociétés en développement ${ }^{1}$. L'institution éducative reflet des contradictions sociales, culturelles et politiques est caractérisée par des réformes permanentes et une hybridation institutionnelles

Si l'on se réfère aux formations institutionnelles éducatives, il importe ainsi de différencier les institutions éducatives qui sont universelles des institutions scolaires qui sont spécifiques à certaines sociétés.

Historiquement les institutions scolaires ont été créées dans les seules sociétés de l'écrit qui renvoient initialement, soit à la compréhension des textes sacrés soit à la constitution d'États administrant leur population à distance. L'école a été ensuite mise en relation avec la formation de compétences, avec la transmission des connaissances, l'éveil des esprits permettant d'apprendre à apprendre. Ce modèle est devenu le point de référence universel.

L'enseignement et la scolarisation peuvent être définis de manière universelle comme des processus institutionnalisés de formation et de transmission des connaissances qui développent des aptitudes, des habitudes et des attitudes. Ils se réalisent au sein d'une institution, l'école, qui se caractérise par plusieurs traits: hiérarchie des cycles de formation, séparation vis-à-vis de la production, corps spécialisé d'enseignants rémunérés, sanction des acquisitions des savoirs par des promotions et par des diplômes. Cette institution fait, en amont, l'objet de choix et de décisions de la part des individus ou des ménages. Elle conduit, en aval, à insérer les élèves sur un marché du travail. (Hugon 2005). Ce processus de formation concerne des individus hétérogènes; il se réalise dans des institutions différenciées, se déroule dans un temps long et se fait dans un univers risqué ou incertain. Cette définition d'une institution "universelle" permet la mesure, la comparaison dans le temps et l'espace des systèmes solaires et de leurs produits ; mais elle présente de nombreuses limites.

Historiquement la colonisation a le plus souvent imposé une école souvent refusée par les élites « indigènes » en opposition avec l'éducation traditionnelle. Mais elle a aussi conduit initialement à un système dualiste proche de l'apartheid entre écoles tronquées pour indigènes et école pour citoyens métropolitains.

Les sociétés ont aujourd'hui des systèmes éducatifs très variés. Les écoles sont religieuses avant d'être républicaines (écoles coraniques, bouddhistes, chrétiennes, juives..). Les "systèmes scolaires » sont éclatés entre des formations d'élites aujourd'hui largement mondialisées par leurs langues, leur mobilités et leurs référents et les formations primaires très différenciées à l'échelle locale privées avec le plus souvent un rôle réduit de l'école républicaine. Sans contrat avec l'État, dans des sociétés à la fois mondialisées et caractérisées par des replis identitaires, on observe le plus souvent une hétérogénéité des filières de formation en relation avec les appartenances sociales, une exclusion de l'école qui s'éloignent fortement du modèle universel de référence

\section{Les liens entre l'institution éducative et le développement}

Nous mettrons à l'épreuve dans cette seconde partie cette économie politique institutionnelle pour analyser plus spécifiquement les liens entre l'institution éducative et le développement. La formation et l'éducation sont considérées dans les travaux des économistes comme un facteur premier du développement avec un rôle accru dans la 
nouvelle économie de l'information et de la connaissance. L'économie mondiale repose de plus en plus sur le savoir. Au sein d'un « capitalisme cognitif » et d'une division cognitive du travail (Delapierre, Moatti, Mouhoud 2000). Or il y a peu de domaines où les enjeux sont aussi conflictuels, que ce soit en termes de valeurs et de connaissances transmises, de tensions entre l'universalisme et le particularisme, d'accès différenciés à l'école selon les catégories sociales ou de divergences entre les États. Après avoir présenté les critères standards de performances de l'institution éducatives (2.1), nous montrerons les limites des mesures de ces performances (2.2), puis développerons une économie politique institutionnelle (2.3).

\subsection{Les critères standards de performance de l'institution éducative}

27 Les questions principales posées par les économistes sont celles de l'efficacité ${ }^{2}$ micro et macro économiques de l'enseignement et celles de l'équité.

Les travaux macro économiques d'après-guerre concernant les institutions éducatives supposées universelles, renvoyaient à des modèles post keynésiens à la Harrod-Domar ou néoclassiques à la Solow (Denison, Poullier 1965) et se situaient dans le cadre de comptabilité nationale reliant les agrégats économiques à des comptes satellites éducatifs. La logique était alors principalement celle de la planification de l'offre scolaire. La performance économique était mesurée en termes de croissance, indicateur unidimensionnel de dimension nationale avec parfois prise en compte des externalités. La méthode d'analyse factorielle, l'analyse en composantes principales, les modèles de croissance endogène permettaient de les effets de l'enseignement sur la croissance avec des rendements croissants conduisant à des processus cumulatifs à la hausse ou à la baisse en deçà de seuils à des trappes à pauvreté (Aghion \& Cohen 2004, Arestoff, Berthelemy 2003).

Les approches micro économiques, de Mincer (1974), Becker (1964) et Schultz (1998) ont assimilé l'enseignement à la formation d'un «capital humain» ayant un taux de rentabilité supposé mesurer sa productivité. L'éducation est du domaine de la valeur d'échange, du commensurable et du calculable. Le capital est mesuré par la somme des flux actualisés des revenus résultant de son usage. L'horizon est celui de revenus futurs anticipés correctement par des agents rationnels. Il y a optimum quand le taux de rentabilité du capital humain égalise le taux d'actualisation. Le lien social peut de même être pris en compte sous la forme du capital social analysé à partir des flux de revenu résultant de son usage

Cette conception du " capital humain ", des liens entre formation et productivité et des taux de rentabilité pour évaluer les effets de l'enseignement indépendamment de l'environnement institutionnel et des différentes formes institutionnelles de l'école est évidemment très discutable pour plusieurs raisons qui ont été développées par les économistes depuis la naissance de l'économie politique. On ne peut supposer des relations immédiates entre formation, productivité et revenu. Il faut différencier les effets productifs des formations spécifiques et génériques. La productivité prend naissance en situation de travail et est valorisée sur un marché du travail, institution qui diffère selon les sociétés. Le produit éducatif est un potentiel dont l'utilisation dépend de la future situation du jeune actif, Dès lors, l'incertitude interdit un calcul économique en terme de probabilité. On ne peut supposer une fongibilité entre les différents " capitaux » (physiques, financiers, humains, sociaux, naturels,...). Le "capital humain» est un 
potentiel de ressources incorporé dans la personne comme le "capital social» est un potentiel de ressources liées aux réseaux sociaux. Ils ne peuvent, sauf de manière métaphorique, être traités comme du capital.

31 Ces critiques ont conduit certains économistes de l'éducation à refuser la conception substantielle du produit éducatif assimilé à l'utilité, à la productivité ou à l'information révélatrice des caractéristiques propres des individus. Selon la théorie du filtre (Arrow, 1973), la formation et le diplôme sont un signal (Spence 1974) qui révèle et certifie les aptitudes individuelles et sert à classer les individus selon leur productivité escomptée. Cette théorie se réfère, en fait, à un contexte d'organisations productives efficientes spécifiques aux sociétés industrielles. Dans la théorie de la segmentation, différenciant marché interne et externe du travail, le diplôme n'est « pas approximation cardinale de la productivité mais indicateur de classement ordinal de l'employabilité » (Vinokur, 1993).

32 La question de la performance éducative s'est ainsi déplacée vers le signal de qualité. Celle-ci est liée à un processus de hiérarchisation où se mêlent des critères d'excellence, de réputation et d'image, de confiance et d'accréditation et de "rating ». Ainsi que l'écrit Annie Vinokur, "le diplôme est un "titre" qui circule dans un espace de confiance déterminé par son émetteur et dont la convertibilité suppose un espace structuré par des systèmes d'équivalence» (2005). Les institutions scolaires sont censées certifier les qualités des savoirs; elles sont elles-mêmes certifiées par des institutions d'évaluation et par des accréditations, certificats d'assurance de la qualité (Vinokur 2005). Cette conception de la qualité fondée sur des caractéristiques objectivables et des aptitudes à répondre à la demande a fait l'objet de nombreuses critiques des courants institutionnalistes . Selon la conception néo institutionnaliste à la North (1990), ce sont les standards de qualité les plus efficients qui tendent à s'imposer dans une concurrence institutionnelle aujourd'hui internationale. Selon les conventionnalistes, la qualité et sa mesure sont fonction des conventions en usage dans l'évaluation et des espaces pluriels de justification (Bailly \& Chatel, 2004).

\subsection{Les limites des mesures des performances des institutions éducatives}

Plusieurs limites apparaissent pour évaluer les performances des institutions éducatives.

Il y a vieux débat sur le produit de la scolarisation ou de l'enseignement (élèves formés, acquisition des connaissances, amélioration des compétences cognitives...), sur les moyens de sa mesure et sur ses effets. L'institutionnalisation de l'éducation par l'école est une manière de contrôler et de connaître à partir de cadres normés et comparables. L' éducation multidimensionnelle et multifonctionnelle, dont la scolarisation n'est qu'une composante insérée dans des systèmes socio historiques spécifiques, est en partie incommensurable. L'éducation est à la fois un processus de formation de la personne et de socialisation, un processus diffus de transmission et de création de savoirs pluriels institués et non institués et de cultures.

Il faut également prendre en compte que les contextes éducatifs sont très hétérogènes Toutes les sociétés connaissent des systèmes éducatifs alors que l'école ne s'est développée qu'en liaison avec l'État et les instances religieuses. Celle-ci a été implantée historiquement en relation avec la religion, la constitution de l'État et souvent par la contrainte. Elle est différemment légitimée selon les sociétés. Elle a ainsi dans les sociétés asiatiques un 
ancrage ancien et profond alors qu'elle est moins intégrée dans la plupart des sociétés africaines. L'école a été refusée historiquement par certains groupes comme forme d'aliénation et d'acculturation ou de remise en cause de leur pouvoir ou des institutions éducatives «traditionnelles ». Le système a été très hétérogène et différemment implanté dans certaines régions. On a observé la mise en place d'un système dual d'apartheid entre citoyens et « indigènes ». Les systèmes n'ont été unifiés sur le plan formel qu'au moment des indépendances mais ils se sont alors constitués en filières éducatives. Le développement de la logique marchande et de la recherche de la performance finit par dicter les actes des individus et leur finalité et la dominance de la mesure correspond à un monde de la démesure (Jany-Catrice 2012).

Alors que le modèle véhiculé par l'UNESCO et appliqué par les États était celui du financement public de l'école et d'une planification de l'offre pour atteindre une scolarisation universelle, l'accent est mis aujourd'hui, dans une approche individualiste, sur la privatisation et sur le rôle de la demande des familles et des firmes. Les classifications conçues dans un cadre étatique et permettant les comparaisons internationales (sous le contrôle de l'UNESCO (Cusso, d'Amico 2005) sont remises en question dans un contexte de pluralité de producteurs d'information, de rôle hégémonique joué par le monde anglo-saxon, par l'OCDE et la Banque mondiale, et de priorités gestionnaires. Les mesures de qualité et de performances éducatives selon des normes de compétitivité, de bonne gestion et de bonne gouvernance l'emportent sur la constitution d'appareils statistiques aux séries temporelles longues, homogènes et fiables.

Enfin, la globalisation a des doubles effets de différenciation et de normalisation. On observe une intensification de la mobilité du capital et du travail qualifié, d'où une disjonction territoriale entre les individus, les systèmes éducatifs et les processus d'accumulation. L'internationalisation $\mathrm{du}$ processus de formation se manifeste, notamment au niveau universitaire, par le déploiement des filiales des grandes maisons mères. Le marché mondial de l'éducation est estimé à deux mille milliards de dollars. Les certifications de savoir acquis que l'école dispensait dans un cadre réglementaire national tendent à céder la place à des évaluations visant à certifier les formations supérieures sur un "marché » mondial, ou du moins régional pour l'Europe, des compétences. Les processus cumulatifs liés aux rendements croissants et aux externalités de la connaissance se traduisent par des divergences internationales entre les trajectoires. Ce processus de standardisation s'accompagne d'un renforcement des disparités des systèmes éducatifs et de concurrence entre des écoles et universités aux statuts pluriels.

\subsection{Vers une économie politique de l'évaluation des performances de l'éducation}

La majorité des approches économiques fait abstraction des contextes socio historiques, des liens entre l'avoir, le savoir et le pouvoir, des systèmes de valeur et des représentations que les populations se font de l'institution scolaire. Les pays pauvres sont ainsi caractérisés le plus souvent à la fois par une sous scolarisation aux différents degrés, une mal scolarisation du fait de la faible qualité moyenne et une sur scolarisation par rapport aux capacités de financement et d'absorption des sortants du système scolaire. L'école importée est plus ou moins appropriée par les populations et est souvent rejetée comme symbole de l'occidentalisation. L'évaluation des performances ne peuvent être 
dissociée du contexte socio historique, de l'enchâssement de l'école dans des rapports sociaux divers et évolutifs et dans des institutions plurielles.

On observe également une hétérogénéité des processus éducatifs et des systèmes scolaires par diversification des filières au sein du champ éducatif et par informalisation, recomposition des frontières entre savoirs institués et non institués. La diversité des formations institutionnelles éducatives oppose les sociétés d'accès ouvert (au sens de North et al.) notamment par l'école aux sociétés de rentes et de reproduction sociale. Dans une conception d'économie politique intégrant les pouvoirs et les conflits, la qualité renvoie au système de valeur imposé par les acteurs hégémoniques réduisant la pluridimensionnalité des critères d'évaluation à leur seule échelle de valeur. La question est alors de savoir qui définit l'excellence, selon quels critères, en fonction de quels moyens financiers, médiatiques ou autres, pour arriver à une reconnaissance par le «marché » des niveaux de diplômes délivrés par les instances éducatives et des compétences reconnues sur le marché du travail qualifié.

L'évaluation des effets de l'enseignement est ainsi largement liée au souci de contrôle de l'État (le terme statistique vient de l'État) et, aujourd'hui, à la marchandisation de la société, notamment au rapport salarial et au marché du travail (Hugon 2013).

41 L'école peut certes jouer un rôle central dans les transformations en profondeur de la société : libération des femmes, développement des facultés cognitives, maitrise de la fécondité, mobilité sociale, passage de sociétés de rentes en sociétés d'innovation... Elle peut favoriser l'innovation et la créativité. Mais, la question de la performance ne peut être dissociée des conflits entre les systèmes de valeurs et des choix politiques propres aux institutions éducatives. L'effet propre de l'enseignement interfère avec d'autres phénomènes et la scolarisation est plus un facteur permissif ou facilitateur qu'une cause.

Nous avons dans la première partie relativisé les critères de performance des institutions scolaires selon différents courants institutionnalismes tout en montrant les limites d'une approche normative privilégiant le "modèle » occidental Tout système éducatif s'insère dans une matrice socioculturelle et sociopolitique. L'éducation, dont la scolarisation n'est qu'une composante, est "avant tout le moyen par lequel la société renouvelle perpétuellement les conditions de sa propre existence " (Durkheim, 1922). Elle est historiquement au cœur de la construction de la citoyenneté et de la nation, du fonctionnement de l'État, de la langue de communication et de diffusion de savoirs spécifiques liées aux diverses cultures. Elle est en même temps un lieu de diffusion de savoirs universels, de maîtrise des mécanismes fondamentaux, d'apprentissage de langues de communication véhiculaires internationales et de connaissances favorisant les compétences et les innovations au sein des unités productives. Les systèmes éducatifs, comme toutes les institutions, sont des systèmes ouverts, éléments en interrelation où interviennent une pluralité de phénomènes, des incertitudes ou des indéterminations (temps non probabiliste), des poly-causalités et des acteurs innovants. Les propriétés dynamiques des systèmes éducatifs sont caractérisées par des innovations, par des processus décentralisés de nouveauté et par des mécanismes collectifs de sélection. L'étude des institutions scolaires implique de prendre en compte leur environnement comme enveloppe des évolutions possibles d'une organisation assujettie à un principe de contingence et de variété. 
43 L'étude des institutions scolaires implique ainsi de prendre en compte leur environnement comme enveloppe des évolutions possibles d'une organisation assujettie à un principe de contingence et de variété.

L'efficacité d'un système éducatif ne peut être isolée du régime d'accumulation et du mode de régulation et de l'insertion de la société dans la mondialisation. D'un côté, les sociétés sont caractérisées par des régimes différents et l'on doit différencier les économies rentières, d'accumulation extensive, d'accumulation intensive et de capitalisme cognitif ou patrimonial. Le rôle des connaissances, du savoir, ne peut être analysé indépendamment de ces régimes. Dans un contexte de mondialisation, les objectifs de compétitivité et de certification des compétences s'imposent. Il y a mise aux normes des sociétés selon des critères internationaux largement télécommandés par les puissances dominantes et par une logique marchande. L'expérience montre que, dans un monde cloisonné, la technologie avancée et les connaissances tacites sont interdites aux populations des pays pauvres. La question éducative déborde aujourd'hui largement le cadre des États nations. L'enseignement s'est mondialisé ou « triadisé ». Les élites formées dans les pays pauvres sont attirées vers les pôles de croissance économique.

En conclusion, le monde se traduit par des asymétries spatiales et des dynamiques inégalitaires croissantes qui interdisent de penser l'uniformisation. Une approche institutionnaliste incorporant l'histoire doit étudier les conflits, les luttes, les contrepouvoirs et voir comment, dans un contexte donné, il y a pluralité des institutions et domination de certaines. Les transitions institutionnelles sont plurielles et permettent d'opposer les processus évolutifs des processus involutifs. Les configurations institutionnelles diffèrent entre les pays émergents, les pays pauvres pris dans des trappes à pauvreté et les États faillis ou en conflits connaissant des processus involutifs. La liaison entre les institutions et le développement et des relations entre le savoir, l'avoir et le pouvoir renvoie aux tensions entre l'universalisme et le particularisme, aux valeurs accordées à l'innovation et à la conservation des patrimoines, et en définitive aux projets politiques et idéologiques des différents acteurs. La question de la mesure et de l'évaluation est ainsi celle de la confrontation des systèmes de valeurs et de l'élaboration d'un processus décisionnel permettant un débat public et un choix politique.

\section{BIBLIOGRAPHIE}

Aghion P. \& Cohen E., (2004) «Éducation et croissance », Note CAE, La Documentation française, $\mathrm{n}$ $\circ, 46$.

Aoki M, (2006), Fondements de l'analyse institutionnelle comparée, Paris, A. Michel, trad MIT 2001

Arrow K.-J., (1973) « Higher education as a filter », Journal of Public Economics, 2, p. 193-216.

Bachelard G., (1973), 4e ed., Essai sur la connaissance approchée, Paris, Vrin.

Bailly F. \& Chatel E, (2004), « Conventions et institutions : approfondissement théorique et contributions au débat politique », Problèmes économiques, n² 2850, août, pp. 24-27. 
Barro L., (1993), “International Comparisons of Educational Attainment ». Journal of Monetary Economics, vol. 32 .

Becker G., 1964, Human Capital. A Theorical and empirical analysis, with special reference to Education Chicago, University of Chicago Press.

Berthelemy J-Cl, Arestoff (F), « Les stratégies d'éducation et de développement en Afrique » in Bauchet $\mathrm{P}$, Germain (P), L'éducation facteur du développement durable en Afrique, Cahier des sciences humaines et politiques, Paris, PUF.

Billaudot B., (2004), « Développement et croissance. Les enjeux conceptuels des débats actuels », communication présentée lors des 1ères journées du développement du GRES 16 et 17 septembre, Université Montesquieu-Bordeaux IV.

Boltanski L., Thevenot L., (1991), De la justification : l'économie des grandeurs, Paris, Gallimard

Boltanski L., Chiapello, (1999), Le nouvel esprit du capitalisme, Paris Gallimard

Boyer R, Saillard Y (eds) (1995), Théorie de la régulation. L'état des savoirs, Paris, La découverte, « Recherches».

Coase R.H (1988), La Firme, le Marché et le Droit, trad Paris Diderot, multimédia 1997

Cusso D’Armico (2005) « Vers une comparabilité plus normative des statistiques internationales de l'éducation : de l'éducation de masse aux compétences ", Cahier de la recherche sur l'éducation et les savoirs, hors-série $\mathrm{n}^{\circ}$ 1,p.21-47

Delapierre M, Moati Ph, Mouhoud E.M. (2000), Connaissance et mondialisation, Paris, Economica Douglas M. (2004) Comment pensent les institutions? Trad, Paris, La découverte.

Denison E.-F. \& Poullier F., 1965, Why Growth rates differ? Postwar experiences in nine western countries, Washington, The Brooking Institution.

Durkheim E., (1922), Education et sociologie, Paris, Alcan.

Favereau O. (1995), « La question du développement et l'économie des conventions « in Hugon Ph., Pourcet G., Quiers Valette S. (1994), L'Afrique des incertitudes, Paris, PUF.

Foucault M (2001), « La gouvernementalité » in Dits et écrits, Paris, Gallimard, t. II.

Greif A. (2006) Institutions and the Path to the Modern Economy Lessson from Medieval Trade, Cambridge, Cambridge University Press.

Hall PA, Taylor RG (1997), « La science politique et les trois néo-institutionnalismes », Revue française de sciences politiques, Vol $47 \mathrm{n}^{\circ}$ 3-4, p. 469-496.

Hugon Ph. (2005) «La mesure des effets économiques de la scolarisation. Apports et limites de l'économie de l'éducation ", $\mathrm{CRES} \mathrm{N}^{\circ} 1$, pouvoir et mesure en éducation.

Hugon Ph (2012), Géopolitique de l'Afrique, Paris SEDES ( $\left.3^{\mathrm{e}} \mathrm{ed}\right)$.

Hugon Ph. (2013), « La mesure du développement est-elle l'expression de rapports de pouvoir? Application au champ de l'éducation » Revue Tiers Monde, n² 213, Janvier-mars p. 105-122

Jany-Catrice F., (2012), La performance totale : nouvel esprit du capitalisme?, Ed du Septentrion.

Lau J., Jamison D. T. \& Louat F. F., (1990) Education and productivity in Developing Countries: An Aggregate production function approach, Working paper WPS 612, Washington.

Lafaye De Michaux E., Ould-Ahmed P. (2007), Les contours d'un projet institutionnaliste en économie du développment. 
Meisel N, Ould-Aoudia J (2008), « La "bonne gouvernance” est elle une bonne stratégie de développement? ", WP AFD N58, janvier.

Mincer J., (1974), Schooling, experience and Earnings, New York, Columbia University Press.

Nelson R., S. Winter (1982), An Evolutionnary Theory of Economic Change Cambridge, Harvard University Press.

North D, R.P. Thomas (1973), The rise of the Western world. A new Economic history, Cambridge, Cambridge university Press.

North D., (1990), Institutions, Institutional Change and Economic Performance, New York, Cambridge University Press.

North D, Wallis Jj, Weingast Br (2006), A conceptual Framework for Interpreting Recorded Human History, NBER WP n 12795, trad Violences et ordres sociaux, Paris, NRF Gallimard, 2010.

Ostrom E., Gardner R., Wolker J. (1994), Rules, Games and Common Pool. An Arbor university, Michigan.

Rodrik D., Subramanian A., Trebbi F. (2004), Institutions Rule: The Primacy of Institutions over Geography and Integration in Economic Development", Journal of Economic Growth, vol. 9-2, pp. 131-165.

Schultz P. (1998), « Comparaison between rate of return of education Ghana and Ivory Cost », miméo, University of Chicago.

Spence M.-A., (1974), Market Signaling; Informational Transfer in Hiring and Related Screening Processes , Harvard U. Press, Cambridge.

Stiglitz J., Sen A., Fitoussi J.-P. (2010), Rapport de la commission sur le mesure de la performance économique du progrès social, 2.

Tallard M., Theret B., Uri D eds (2000), Innovations institutionnelles et territoires, Paris, L'Harmattan.

Vinokur A., (2005), « La mesure de la qualité des services de l'enseignement. Construction des marchés transnationaux, gouvernance de l'instruction », CRES, hors série nº 1, pp. 83-108.

Williamson O. (2000), « The New institutional Economics. Taking Stock, Looking Ahead » Journal of Economic Litterature vol XXXVIII, sept 595- 613.

Yifu Lin J. (1989), « An economic Theory of Institutional Change. Induced and Imposed Change » Cato Journal, $\mathrm{N}^{\circ} 1$, vol. 9, 1-33.

Yong H. (1994), « économie néo-institutionnelle et développement. Une analyse synthétique », Revue d'économie du Développement, $\mathrm{n}^{\circ}$ 4, pp 125-14.

\section{NOTES}

1. Comme l'analyse Ostrom et al (1994), les droits d'appropriation (property rights) sont composés de droit d'accès (access right), de droit de prélever, de soustraire, d'extraire (withdrawal right) une et/ ou plusieurs ressources, de droit de gérer (management right), de droit d'exclure (exclusion right), de droit d'aliéner et/ ou droit de transfert (alienation right). Les combinaisons sont multiples. Des gestions de biens communs (bornes fontaines, puits) sont possibles, avec contributions des consommateurs, moyennant des règles évitant la "tragédie des biens communs" (cf. la critique de Hardin par Ostrom J 1994). 
2. L'efficacité se mesure à l'aune des comparaisons des effets réels aux objectifs fixés. L'efficience suppose un bilan coût/avantages. La question de l'efficacité des dépenses d'éducation et de leur financement renvoie à l'allocation optimale des ressources à l'intérieur du système scolaire (efficacité interne) et entre le système scolaire et le système économique (efficacité externe). Mesurer l'efficience et l'efficacité de l'enseignement suppose que les objectifs soient explicités de la part des décideurs (efficacité) et que les coûts et les avantages soient évalués au regard d'échelles de valeurs révélées.

\section{RÉSUMÉS}

Les institutions diffèrent selon les sociétés et les critères de performances sont pluriels. Les économies en développement sont des formations institutionnelles caractérisées par des institutions endogènes et exogènes avec plus ou moins endogénéisation. Cet article présente une économie politique institutionnelle prenant en compte la pluralité des profils institutionnels, puis analyse plus spécifiquement les liens entre les institutions éducatives et le développement économique.

The institutions differ according to the countries and the criteria of performances are plural. The economies under development are institutional formations characterized by endogenous and exogenic institutions with more or less endogeneisation. This article presents an institutional political economy taking of account the plurality of the institutional profiles, then analyzes more specifically the links between the education institutions and economic development.

\section{INDEX}

Mots-clés : développement, économie politique institutionnelle, éducation, profils institutionnels, performance

Keywords : development, political economy, education, trainings, institutional profiles, performance

Code JEL I21 - Analysis of Education, I32 - Measurement and Analysis of Poverty

\section{AUTEUR}

\section{PHILIPPE HUGON}

Université Paris Ouest Nanterre-La Défense 